\title{
Large dose hyperpolarized water with dissolution-DNP at high magnetic field
}

\author{
Lipsø, Hans Kasper Wigh; Bowen, Sean; Rybalko, Oleksandr; Ardenkjær-Larsen, Jan Henrik
}

Published in:

Journal of Magnetic Resonance

Link to article, DOI:

10.1016/j.jmr.2016.11.008

Publication date:

2016

Document Version

Peer reviewed version

Link back to DTU Orbit

Citation (APA):

Lipsø, H. K. W., Bowen, S., Rybalko, O., \& Ardenkjær-Larsen, J. H. (2016). Large dose hyperpolarized water with dissolution-DNP at high magnetic field. Journal of Magnetic Resonance, 274, 65-72.

https://doi.org/10.1016/j.jmr.2016.11.008

\section{General rights}

Copyright and moral rights for the publications made accessible in the public portal are retained by the authors and/or other copyright owners and it is a condition of accessing publications that users recognise and abide by the legal requirements associated with these rights.

- Users may download and print one copy of any publication from the public portal for the purpose of private study or research.

- You may not further distribute the material or use it for any profit-making activity or commercial gain

- You may freely distribute the URL identifying the publication in the public portal

If you believe that this document breaches copyright please contact us providing details, and we will remove access to the work immediately and investigate your claim. 


\section{Title page}

Title:

Large dose hyperpolarized water with dissolution-DNP at high magnetic field

\section{Authors:}

Kasper Wigh Lips $\varnothing^{1}$, Sean Bowen ${ }^{1}$, Oleksandr Rybalko ${ }^{1}$ and Jan Henrik Ardenkjær-Larsen ${ }^{1,2}$

\section{Affiliations:}

${ }^{1}$ Department of Electrical Engineering, Technical University of Denmark, Kgs. Lyngby, Denmark.

${ }^{2} \mathrm{GE}$ Healthcare, Brøndby, Denmark.

\section{Corresponding author and Reprint info:}

Jan Henrik Ardenkjær-Larsen, DTU Electrical Engineering, Ørsteds Plads, Building 349, room 126, 2800 Kgs. Lyngby, Denmark. Tel (0045) 4525 3918. E-mail: jhar@elektro.dtu.dk

\section{Grant support:}

This work was supported by the Danish Research Council [grant number 12-127232] and the Danish National Research Foundation [grant number DNRF124].

The authors confirm that this article content has no conflict of interest.

Keywords: hyperpolarized water; dissolution-DNP; hyperpolarization 
[Type here]

\begin{abstract}
We demonstrate a method for the preparation of hyperpolarized water by dissolution Dynamic Nuclear Polarization at high magnetic field. Protons were polarized at $6.7 \mathrm{~T}$ and $1.1 \mathrm{~K}$ to $>70 \%$ with frequency modulated microwave irradiation at $188 \mathrm{GHz} .97 .2 \pm 0.7 \%$ of the radical was extracted from the sample in the dissolution in a two-phase system. $16 \pm 1 \mathrm{~mL}$ of $5.0 \mathrm{M}^{1} \mathrm{H}$ in $\mathrm{D}_{2} \mathrm{O}$ with a polarization of $13.0 \pm 0.9 \%$ in the liquid state was obtained, corresponding to an enhancement factor of 4,000 \pm 300 compared to the thermal equilibrium at $9.4 \mathrm{~T}$ and $293 \mathrm{~K}$. A longitudinal relaxation time constant of $16 \pm 1 \mathrm{~s}$ was measured. The sample was polarized and dissolved in a fluid path compatible with clinical polarizers. The volume of hyperpolarized water produced by this method enables angiography and perfusion measurements in large animals, as well as NMR experiments for studies of e.g. proton exchange and polarization transfer to other nuclei.
\end{abstract}


[Type here]

\section{Introduction}

NMR is an important analytical tool for e.g. structure analysis, chemical quantification, and investigation of biochemical pathways. Similarly, MRI is a valuable and safe diagnostic tool in medical applications. However, both techniques share a key weakness - poor sensitivity caused by low polarization of nuclear spins. Through the use of hyperpolarization, the signal can be enhanced by several orders of magnitude; leading directly to an increase in sensitivity. This increase in signal obviates the need for time-consuming signal averaging, permitting real-time experiments with unprecedented time resolution. An especially promising application of this capability is the study of chemical and biochemical reactions both in vitro and in vivo.

The field of dissolution-Dynamic Nuclear Polarization (d-DNP) has developed rapidly since the first demonstration in 2003 [1]. The main focus has been on hyperpolarization of ${ }^{13} \mathrm{C}$ nuclei for metabolic imaging, where preclinical studies of hyperpolarized ${ }^{13} \mathrm{C}$ labelled pyruvic acid was injected for characterization of cancer [2-4] or other metabolic diseases in animal models [5,6]. Hyperpolarization has also been used for angiographic and perfusion imaging [7-9] with agents that are not metabolically active. Concurrently, hyperpolarized ${ }^{13} \mathrm{C}$ has been applied for signal enhancement in NMR experiments, such as kinetic studies of metabolism [10-12] or structure analysis of biochemical compounds $[13,14]$.

In recent years, the interest in hyperpolarization of protons diluted in heavy water has increased $[15,16]$. Due to the high gyromagnetic ratio of the proton and the high proton concentration in water, the achievable magnetization may exceed what is possible with hyperpolarized ${ }^{13} \mathrm{C}$ or other nuclei. Furthermore, hyperpolarized proton imaging is facilitated by already existing MRI coils and sequences, in contrast to hyperpolarized ${ }^{13} \mathrm{C}$ that requires non-standard equipment. In one approach, water, polarized by the Overhauser effect at $0.35 \mathrm{~T}$, was applied for MRI $[17,18]$. However, the Overhauser effect typically achieves an enhancement of 100 times at $0.35 \mathrm{~T}$ for pure water, corresponding to a polarization of only $0.001 \%$. Secondly, the short $T_{1}$ of pure water, $3.6 \mathrm{~s}$, limits the available time window significantly. Both of these limitations can be overcome by d-DNP. A 3.35 T HyperSense polarizer was used to produce angiographic images in the rat venous system and head with ${ }^{1} \mathrm{H}$ polarization of $3.5 \%$ and ${ }^{1} \mathrm{H}$ concentration of 3.9 $\mathrm{M}$ [19], and hyperpolarized water has been used for signal enhancement in NMR experiments $[20,21]$. Hyperpolarized protons have been applied to enhance the inherent low sensitivity of larger biomolecules, and proton exchange has facilitated increased sensitivity leading to large reduction in scan time [22], or to accommodate studies of protein-ligand interactions for drug discovery [23]. Polarization, dissolution and radical extraction for water/DMSO- $d_{6}$ samples was shown to give polarization of 
[Type here]

approximately $25 \%$ and $T_{1}>40 \mathrm{~s}$ [24]. $6 \%$ proton polarization and $T_{1}$ of $>36 \mathrm{~s}$ has been demonstrated by attaching nitroxide radicals to polymer-based backbones that could be filtered out at dissolution [25].

In this work we study the hyperpolarization of ${ }^{1} \mathrm{H}$ in $\mathrm{D}_{2} \mathrm{O}$ with $\mathrm{d}$-DNP at $6.7 \mathrm{~T}$ and $1.2 \mathrm{~K}$ using the fluid path of [26]. The aim of the study was to investigate the polarization and dissolution at these experimental conditions.

\section{Theory}

DNP relies on the presence of electron spins, from which polarization is transferred to nuclear spins by microwave (MW) irradiation. An increase in performance has recently been demonstrated when the MW field is frequency modulated $[27,28]$. The unpaired electron spins are typically added to the system in the form of stable organic radicals such as nitroxides or trityls. Paramagnetic species, such as radicals, efficiently promote nuclear spin relaxation, yet they are a key requirement in the DNP process. A key challenge in dissolution-DNP is supplying sufficient paramagnetic agent to perform DNP, without negatively influencing the relaxation properties of the sample after dissolution. This trade-off is more pronounced due to the high gyromagnetic ratio of ${ }^{1} \mathrm{H}$ that renders it more susceptible to paramagnetic relaxation. Hence, the radical should be segregated from the nuclei upon serving its purpose.

Nitroxides are well suited for DNP of protons due to their broad electron paramagnetic resonance (EPR) lines [29]. With a reported relaxation rate contribution of $0.55 \mathrm{~s}^{-1} \mathrm{mM}^{-1}$ at $25^{\circ} \mathrm{C}$ in low field [30], the dissolved sample would lose the enhanced polarization in a few seconds with the radical present. Thus, radical extraction is a crucial element for achieving long $T_{1}$ and, ultimately, high polarization after the dissolution and transfer process. Small animal angiography with hyperpolarized water has been demonstrated in a $3.35 \mathrm{~T}$ polarizer [19], where the radical induced relaxation was eliminated by scavenging with ascorbic acid, as demonstrated earlier in [31]. However, the ascorbic acid needs to be hundreds of $\mathrm{mM}$ to quench the radical effectively, and the quenching process takes a significant amount of time, during which relaxation occurs. Furthermore, the ascorbic acid undergoes thermal degradation that leads to browning during the solvent heating process, complicating the process further. Hence, we describe a system where the nonpolar nitroxide radical is rapidly extracted into an organic phase immediately after dissolution, as previously demonstrated in [32].

In addition, the $T_{1}$ is sensitive to the proton concentration. A high proton concentration will enhance the final magnetization, but will concurrently increase the dipolar relaxation between proton nuclei, and thus cause faster relaxation. A proton concentration of $5.0 \mathrm{M}$ is determined to achieve a high signal in the injectable sample, giving rise to a relaxation rate contribution of less than $0.04 \mathrm{~s}^{-1}$ at $21.5^{\circ} \mathrm{C}$ [33]. A final 
[Type here]

relaxation contributor is the temperature, where a warmer sample will have higher proton diffusivity, and thus retain polarization longer, within the relevant temperature range [34].

Rapid dissolution and radical extraction are crucial for maintaining a high solution state polarization. We accelerate the dissolution by addition of Nonafluorobutyl methyl ether (NFBME) with boiling point of $60^{\circ} \mathrm{C}$ and density $\rho=1.529 \mathrm{~g} / \mathrm{mL}$ to the dissolution medium syringe. The low boiling point results in a high pressure to drive the flow in the fluid path, and the density ensures that the sample will be dissolved and diluted in $\mathrm{D}_{2} \mathrm{O}$ rapidly, and that the denser NFBME will follow, due to the vertically aligned, upwards facing syringe. Another feature of addition of an immiscible solvent in the dissolution medium syringe is reduction of variation in the volume reaching the sample, because all dissolution medium is flushed out of the tubes, while the dead volume consists of the organic solvent only.

The oxygen in the system originating from air in the fluid path and dissolved in the solvents contributes to the relaxation rate with $0.0094 \mathrm{~L} / \mathrm{mg} / \mathrm{s}$ [35]. The concentration of dissolved oxygen in air saturated water at $25^{\circ} \mathrm{C}$ is around $8.6 \mathrm{mg} / \mathrm{L}$, which leads to a relaxation rate contribution of $0.081 \mathrm{~s}^{-1}$. The corresponding oxygen concentrations in heptane and NFBME, are a factor of 10 and 20 higher, respectively [36,37]. The system thus requires degassing of the solvents, as well as flushing of the entire fluid path and receiver system with inert gas.

Radiation damping: The precession of the transverse magnetization of the protons after excitation induces a current in the NMR coil that contributes to restoring the magnetization to the longitudinal direction. Considering the large magnetization of hyperpolarized water protons, this mechanism will have a significant effect on the transverse relaxation rate. In addition to the magnetization, the contribution to the reduced time constant of the free-induction-decay (FID) is affected by parameters such as the Q-factor and the filling factor of the coil [38], or it can be treated by the reciprocity principle [39]. The observable transverse relaxation time $T_{2}^{o b s}$ is given by $\frac{1}{T_{2}^{o b s}}=\frac{1}{T_{2}^{*}}+\frac{1}{\tau_{R D}}$, where $T_{2}^{*}$ is the time constant of the FID in the absence of radiation damping (typically due to field inhomogeneity) and $\tau_{\mathrm{RD}}$ is the radiation damping time constant. We show that the radiation time constant, $\tau_{\mathrm{RD}}$, can be used as a measure of polarization, with a series of concentration dependent, thermally polarized radiation damping time constants as reference. The method complements quantification of polarization by comparison of integrals for spectra that are hyperpolarized and at thermal equilibrium.

\section{Methods}

DNP: All experiments were performed using a homebuilt DNP polarizer (6.7 T magnet from Magnex, Oxford, UK) with a flow-type variable temperature insert. The DNP probe was conceptually based on the 
[Type here]

idea introduced in 2003 [1]. Microwaves were provided by a microwave source VCOM-10/94-WPT (ELVA-1, St. Petersburg, Russia) and a 200X2R4 frequency doubler (VDI, Charlottesville, VA, USA), which provided an output power of $55 \mathrm{~mW}$ at $188 \mathrm{GHz}$. The source had a tuning range of $\pm 0.6 \mathrm{GHz}$. The output power after transmission through the $800 \mathrm{~mm}$ circular waveguide and the microwave window [40] was measured to $5 \pm$ $1 \mathrm{~mW}$ using a horn and an Ericsson power meter (VDI, Charlottesville, VA, USA). Solid state NMR was performed with a saddle coil formed around a polytetrafluoroethylene (PTFE) former connected to a nonmagnetic coaxial cable (type Stainless Steel 304/Beryllium Copper, SCB Service and Consulting, Neuss, Germany) and a tune and match box. NMR measurements were performed with a Varian INOVA console (Palo Alto, CA, USA). The polarizer was fitted with a heater-pressure module, airlock and insertion module to allow use of the fluid path (both described in [26]). The microwave frequency was sinusoidally swept with a rate of $f_{\text {mod }}$ and amplitude $a_{\text {mod. }}$. The samples were polarized for $60 \mathrm{~min}$.

Solid state NMR: The build-up of polarization was monitored by acquisition of NMR spectra every $120 \mathrm{~s}$ from initiation of MW irradiation. A pulse of $1 \mu \mathrm{s}$ at $2.7 \mathrm{~W}$ corresponding to a flip angle of approximately $0.3^{\circ}$ was used for excitation, and 1024 complex points with a spectral width of $5 \mathrm{MHz}$ were acquired. Short MW frequency sweeps were measured with the same parameters, except only with 60 s between each MW frequency step and increased receiver gain. The magnetization was saturated before each MW frequency step by a train of 3000 pulses of $30^{\circ}$ flip angle. Long MW frequency sweeps were monitored for one hour at each MW frequency. The NMR signal was destroyed by a train of saturating pulses before each build-up (MW frequency), and then measured every $120 \mathrm{~s}$. Similar for long MW power sweeps. The build-up curve for each MW frequency or power setting was fitted to a mono-exponential function to obtain asymptotic value and time constant. Polarization was quantified by allowing a $30 \mathrm{mM}$ water/glycerol sample to polarize towards thermal equilibrium in the magnetic field at $1.12 \mathrm{~K}$ over $19 \mathrm{~h}$, and fitting of the build-up curve of spectra acquired every $300 \mathrm{~s}$. The background signal from the DNP probe was measured with an empty sample cup and subtracted from the data to determine the thermal signal from the sample. $100 \mathrm{kHz}$ exponential line broadening was applied to the data, which were analyzed in MATLAB (Mathworks, Natick, MA, USA).

Sample preparation: Solutions of 20 and 30 mM TEMPO (2,2,6,6-Tetramethylpiperidine 1-oxyl, 98\%, Sigma Aldrich, Denmark) in $\mathrm{H}_{2} \mathrm{O} /$ glycerol (both Sigma Aldrich, Denmark) 1:1 (w/w), and $30 \mathrm{mM}$ TEMPO in $\mathrm{H}_{2} \mathrm{O} /$ DMSO- $\mathrm{d}_{6}$ (Dimethyl sulfoxide-d6, Sigma Aldrich, Denmark) 1:1 (w/w), was prepared. Dissolution medium consisted of $\mathrm{D}_{2} \mathrm{O}$ (99.90\%, Euriso-top, Saint-Aubin, France) with $1 \mathrm{mM}$ calcium disodium ethylenediaminetetraacetic acid (EDTA) and $9 \mathrm{~g} / \mathrm{L} \mathrm{NaCl}$. Sodium chloride was added to provide an isotonic solution. 
[Type here]

Fluid path filling: The fluid path consists of a syringe, two concentric tubes leading to a sample vial and an exit tube. The dissolution medium is heated in the syringe, and transferred via the inner tube to the sample vial for dissolution of the sample, before being flushed back to the syringe via the outer lumen and a threeway valve. The exit tube leads the dissolved sample from the syringe valve to a receiver.

The $1100 \mathrm{mg}$ ( 1 $\mathrm{mL}$ ) sample was injected into the sample vial by the large sample filling method introduced in [41]. This procedure resulted in actual sample mass of $1099.8 \pm 12.6 \mathrm{mg}(N=5) .7 .6 \mathrm{~g}(5 \mathrm{~mL})$ NFBME ( $\geq 99 \%$, Sigma Aldrich, Denmark) and $17.72 \mathrm{~g}(16 \mathrm{~mL}$ ) dissolution medium was filled in the syringe and degassed by bubbling with helium gas for 10 minutes by inserting a tube through an adapter that led the moist gas away from the fluid path openings. In experiments without NFBME, another $12 \mathrm{~mL}$ of dissolution medium was added. The fluid path syringe was closed off before freezing the sample vial in liquid nitrogen for 2 minutes to avoid cryo pumping of moisture into the fluid path. Samples to be degassed were exposed to vacuum and sealed off before melting and sonicating the sample vial. The sample was frozen again; the cycle was repeated three times (freeze-pump-thaw). After freezing, the fluid path was flushed with helium gas for 2 minutes to remove air and provide a helium atmosphere. The sample vial was rapidly transferred from the liquid nitrogen bath to the polarizer.

Dissolution: The syringe was inserted into a heater/pressure module, where the dissolution medium and NFBME were heated to a temperature of $130^{\circ} \mathrm{C}$ over $30 \mathrm{~min}$ at 2.75 bar. The sample was raised $140 \mathrm{~mm}$ out of the helium bath before the dissolution medium syringe three-way valve was switched, releasing the piston in the syringe driven with 16 bar pressure. The $50 \mathrm{~cm}$ outlet tube was inserted into a closed $150 \mathrm{~mL}$ separatory funnel containing $25 \mathrm{~mL}$ degassed heptane ( $\geq 99 \%$, Sigma Aldrich, Denmark), which was shaken vigorously during dissolution and a further $10 \mathrm{~s}$. The aqueous phase was drawn into a syringe, and $1.5 \mathrm{~mL}$ was rapidly injected into an injection line entering a $5 \mathrm{~mm}$ NMR tube situated in the NMR spectrometer, which had been flushed with helium gas and sealed before the experiment. The dead volume of the injection line was $0.6 \mathrm{~mL}$. Temperature was measured with an RTD thermometer (OMEGA HH804U, Stamford, CT, USA) within $30 \mathrm{~s}$ from dissolution.

Liquid state NMR: NMR measurements of the dissolved sample were performed on a $400 \mathrm{MHz}$ spectrometer (Agilent, Palo Alto, CA). A series of 200 spectra were acquired at 1 s intervals with 31200 points, sweep width of $50 \mathrm{kHz}$ and a $1^{\circ}$ flip angle of pulse length $1 \mu \mathrm{s}$ at approximately $0.4 \mathrm{~W}$. The integral was measured as the first point in the FID, and the decay was fitted to estimate the radiation damping time constant. A spectrum of the fully relaxed sample with identical acquisition parameters was measured to quantify the enhancement factor, and thus polarization. The longitudinal relaxation time constant, $T_{1}$, was obtained from the decay of the hyperpolarized signal. The $T_{1}$ for the same sample was subsequently 
[Type here]

confirmed by measuring the NMR signal build-up at 9.4 T when quickly inserting the nonpolarized (earth field) sample. from the build-up of the magnetization after relaxation outside the magnet. Quantitative NMR measurements were performed to determine the final proton concentration in the dissolved sample. $0.2 \mathrm{M}$ potassium formate in $\mathrm{D}_{2} \mathrm{O}$ was added for reference. With the probe used, this concentration did not significantly change the $90^{\circ}$ flip angle. Data were analyzed using VnmrJ (Agilent Technologies, Palo Alto, CA, USA) and MATLAB (Mathworks, Natick, MA, USA).

Radiation damping in thermal experiments: Solutions of equally distributed water $/ D_{2} \mathrm{O}$ volume ratios from $10 \%$ to $100 \%$, corresponding to $11.08 \mathrm{M}$ to $110.82 \mathrm{M}$, were prepared. A series of $10 \mathrm{NMR}$ experiments with parameters identical to those of the hyperpolarized experiments was measured, and the linewidth was determined.

Optical absorbance measurements: The final concentration of TEMPO radical was determined by optical absorbance measurements with an Ocean Optics DH-2000 Deuterium-Halogen light source and an Ocean Optics USB2000+UV-VIS spectrometer in quartz cuvettes of $10 \mathrm{~mm}$ path length. The absorbance at $\lambda=240$ $\mathrm{nm}$ was compared to a reference curve of TEMPO in water at 125, 250, 375 and $500 \mu \mathrm{M}$.

Clinical polarizer compatibility: Organic solvents tend to weaken the fluid path syringe when heated for longer periods, as when inserted in clinical polarizers. To avoid syringe damage during these experiments, the protocol was modified by leaving out the NFBME. Additional dissolution medium was added to a total volume of $28.0 \mathrm{~mL}$. Experiments were performed on a 5 T SPINlab (GE Healthcare, Brøndby, Denmark).

\section{Results}

The solid state polarization from an $1100 \mathrm{mg} 30 \mathrm{mM}$ TEMPO in $\mathrm{H}_{2} \mathrm{O} /$ glycerol 1:1 (w/w) sample was measured (Figure 1), and showed a $T_{1}$ of $583 \pm 62 \mathrm{~min}$. The asymptotic value corresponds to a thermal polarization of $0.61 \%$, and was used for quantification of the DNP enhanced polarization.

Short MW frequency sweeps for three different samples are shown in Figure 2. The polarization of the 30 mM TEMPO in water/glycerol 1:1 (w/w) sample in Figure 2a increases by $30 \%$ at the optimal MW frequency and modulation amplitude of $50 \mathrm{MHz}$. The maximum of the DNP profile left shifts for increasing modulation amplitude, as the MW frequencies giving rise to negative polarization are irradiated. A similar picture is seen for a $20 \mathrm{mM}$ sample (Figure 2b). However, the lower concentration appears to benefit from larger modulation amplitudes. This trend is even more pronounced for samples using DMSO- $\mathrm{d}_{6}$ as glassing agent, Figure 2c. An increase in polarization at optimum MW frequency is observed up to $a_{\bmod }$ of $125 \mathrm{MHz}$. The 
[Type here]

effect of frequency modulation is more pronounced for this sample. Without modulation, the DNP enhancement is significantly lower.

A long MW frequency sweep without frequency modulation is shown in Figure 3, for the $30 \mathrm{mM}$ TEMPO in glycerol/water 1:1 (w/w) sample. The polarization resembles the short build-ups of Figure 2a. However, the optima are shifted towards the center in short sweeps due to the faster build up in the central region ( $\tau_{\mathrm{DNP}}$ curve).

A long power sweep is shown in Figure 4. The asymptotical maximum polarization is reached for $30 \mathrm{~mW}$ power. The DNP polarization after $1 \mathrm{hr}$ increases linearly with the available MW power and the DNP buildup time constant decreases with MW power with a square-root of power dependence. The linear relation between MW power and polarization is valid over the entire DNP spectrum, Figure 5, i.e. the data confirms that build-up rate constant is independent of MW frequency.

The build-up curves of several sample compositions have been measured to study the radical concentration, removal of dissolved oxygen and glassing matrix. The build-up time constants and polarizations after 60 min DNP are presented in Table 1.

\begin{tabular}{|l|l|l|l|}
\hline Experiment & $\boldsymbol{N}$ & $\boldsymbol{P ( 1 ~ h r ) ~ [ \% ] ~}$ & DNP time const. \\
\hline $20 \mathrm{mM}$ TEMPO in water/glycerol at $188.12 \pm 0.05 \mathrm{GHz}$ & 6 & $48.1 \pm 4.9$ & $37 \pm 5 \mathrm{~min}$ \\
\hline $30 \mathrm{mM}$ TEMPO in water/glycerol at $188.12 \pm 0.05 \mathrm{GHz}$ & 4 & $63.1 \pm 3.7$ & $24 \pm 4 \mathrm{~min}$ \\
\hline Degassed $30 \mathrm{mM}$ TEMPO in water/glycerol at $188.06 \pm 0.05 \mathrm{GHz}$ & 5 & $71.5 \pm 4.5$ & $23 \pm 2 \mathrm{~min}$ \\
\hline Degassed $30 \mathrm{mM}$ TEMPO in water/DMSO- $\mathrm{d}_{6}$ at $188.06 \pm 0.05 \mathrm{GHz}$ & 3 & $42.8 \pm 1.7$ & $41 \pm 2 \mathrm{~min}$ \\
\hline
\end{tabular}

Table 1: DNP results for various sample compositions and polarization parameters.

The dissolution resulted in $16 \pm 1 \mathrm{~mL}(N=8)$ aqueous phase entering the heptane bath, to reach a common temperature of $50 \pm 2{ }^{\circ} \mathrm{C}(N=6)$. The total time of dissolution, extraction and transferring of sample from separatory funnel via syringe and tubing to acquisition in NMR spectrometer was less than $25 \mathrm{~s}$. The first spectrum is shown in Figure 6a. The water peak has a line width of $1166 \mathrm{~Hz}$ due to the radiation damping caused by the large magnetization of the sample. The peak of the glycerol aliphatic protons is apparent in the spectrum, indicating that these protons are hyperpolarized along with the water protons.

In this particular experiment, we achieved an enhancement factor of 3,761 at 9.4 T, corresponding to a polarization of $12.1 \%$. A signal time series is shown in Figure 7. The estimated $T_{1}$ after correction for signal loss from previous excitations is $17.0 \mathrm{~s}$. The relation between linewidth (full with at half maximum, FWHM) due to radiation damping and water concentration was found to be $F W H M\left(c_{W}\right)=0.0691 \mathrm{~Hz} \cdot c_{W}+1.92 \mathrm{~Hz}$, where $c_{W}$ is the proton concentration in the sample (Figure $6 \mathrm{~b}$ ). Thus, the linewidth of $1,166 \mathrm{~Hz}$ 
[Type here]

corresponds to an polarization of $11.7 \%$, when corrected for the $111 \mathrm{M}$ proton concentration in water and the $4.6 \mathrm{M}$ proton concentration in the dissolved sample, in close agreement with the time domain integral estimate. The thermal signal and $T_{1}$ was measured by acquiring the NMR signal from the sample as it was introduced into the 9.4 $\mathrm{T}$ magnet, Figure 7 . The measurement was done $5 \mathrm{~min}$ after dissolution when the sample was fully relaxated. A consistent $T_{1}$ of $16.6 \mathrm{~s}$ was measured.

The polarizations and $T_{1}$ 's in the liquid state at $9.4 \mathrm{~T}$ for several samples are shown in Table 2 . The $30 \mathrm{mM}$ TEMPO in water/glycerol 1:1 (w/w) sample showed large fluctuations in the observed polarization, with an average of $7.8 \%$. The polarization was significantly lower when the NFBME was omitted, where the standard fluid path exit tube set-up yielded polarizations below $3 \%$. However, a significant increase in polarization was achieved when the exit tube was reduced from $210 \mathrm{~cm}$ to $50 \mathrm{~cm}$ - a setup that requires minor modifications of a SPINlab. Shortening the exit tube increased the temperature in the dissolved sample with approximately $10^{\circ} \mathrm{C}$, which we believe to be the main reason for the increased polarization. In addition, the shorter time from dissolution to extraction may also play a role.

Larger enhancements were achieved when the $30 \mathrm{mM}$ TEMPO in water/glycerol 1:1 (w/w) sample was degassed in the fluid path before polarization, which can be attributed to the increased solid state polarization (Table 1). However, the degassing only increased the solid state polarization by $13 \%$, whereas the liquid state polarization increased by more than $50 \%$. The amount of oxygen removed from the sample has insignificant impact on the liquid state relaxation rate, and no further $T_{1}$ prolongation was observed.

The liquid state polarization of the degassed water/DMSO- $d_{6} 1: 1(w / w)$ sample was $7.2 \%$, significantly lower than the degassed water/glycerol 1:1 (w/w) sample of $13.0 \%$. The difference is explained by the significantly lower solid state polarization of the water/DMSO- $\mathrm{d}_{6}$ sample.

The method was tested with a sample of half size $(550 \mathrm{mg})(30 \mathrm{mM}$ TEMPO in water/glycerol 1:1 (w/w)) dissolved in half the volume of dissolution medium $(8 \mathrm{~mL})$ and the standard $5 \mathrm{~mL}$ NFBME. Polarization of $12 \%$ and $T_{1}$ of $17.0 \mathrm{~s}$ confirm that the method scales to smaller samples.

\begin{tabular}{|l|l|l|l|}
\hline Experiment & $\boldsymbol{N}$ & Pol. [\%] & $\boldsymbol{T}_{\mathbf{1}}[\mathbf{s}]$ \\
\hline $20 \mathrm{mM}$ TEMPO in water/glycerol 1:1 (w/w) & 5 & $8.6 \pm 1.5$ & $16.1 \pm 1$ \\
\hline $30 \mathrm{mM}$ TEMPO in water/glycerol 1:1 (w/w) & 6 & $7.8 \pm 3.3$ & $17.0 \pm 0.9$ \\
\hline Degassed $30 \mathrm{mM}$ TEMPO in water/glycerol 1:1 (w/w) & 3 & $13.0 \pm 0.9$ & $15.7 \pm 1.2$ \\
\hline Degassed $30 \mathrm{mM}$ TEMPO in water/DMSO-d 6 1:1 (w/w) & 2 & $7.2 \pm 1.3$ & $18.8 \pm 1.6$ \\
\hline $30 \mathrm{mM}$ TEMPO in water/glycerol 1:1 (w/w) without NFBME, long exit tube & 2 & $2.4 \pm 0.6$ & $19.6 \pm 0.8$ \\
\hline $30 \mathrm{mM}$ TEMPO in water/glycerol 1:1 (w/w) without NFBME, short exit tube & 2 & $4.8 \pm 0.5$ & $20.1 \pm 1.3$ \\
\hline
\end{tabular}




\begin{tabular}{|l|l|l|l|}
\hline SPINlab polarization & $4^{*}$ & $5.3 \pm 0.9$ & $24.1 \pm 1.0$ \\
\hline
\end{tabular}

The partition coefficient of TEMPO in water/heptane, water/NFBME and water/[NFBME/heptane 1:3 (V/V)] were similar. The scheme of extraction in the separatory funnel was investigated by a series of dissolutions with varying shaking time. An equilibrium was observed for approx. $5 \mathrm{~s}$ vigorous shaking, and $10 \mathrm{~s}$ shaking time was chosen to ensure complete equilibrium in all experiments. With $10 \mathrm{~s}$ shaking in $25 \mathrm{~mL}$ heptane, we observed a final TEMPO concentration of $50 \pm 12 \mu \mathrm{M}$ (30 mM TEMPO in water/glycerol 1:1 (w/w), $N=5$ ) and $35 \pm 6 \mu \mathrm{M}$ (20 mM TEMPO in water/glycerol 1:1 (w/w), $N=5)$, corresponding to relaxation rate contributions of $R_{30 \mathrm{mM}}=0.028 \mathrm{~s}^{-1}$ and $R_{20 \mathrm{mM}}=0.019 \mathrm{~s}^{-1}$, respectively.

The average final proton concentration in the dissolved glycerol/water 1:1 $(\mathrm{w} / \mathrm{w})$ sample was measured to $5.0 \pm 0.5 \mathrm{M}(N=18)$, compared to the calculated value of $4.6 \mathrm{M}$. The proton concentration of the water/DMSO- $d_{6}$ sample was not measured, but should be $3.6 \mathrm{M}$ if full recovery is assumed.

\section{Discussion and Conclusion}

The presented method enables experiments where large quantities of hyperpolarized water protons are required, e.g. imaging experiments in large animals. We demonstrate $13 \%{ }^{1} \mathrm{H}$ polarization and $T_{1}$ of $16 \mathrm{~s}$ for a $16 \mathrm{~mL}$ sample with $5 \mathrm{M}$ protons in $\mathrm{D}_{2} \mathrm{O}$ on a polarizer operating at $6.7 \mathrm{~T}$ and $1.2 \mathrm{~K}$. This is enabled by the fluid path that accommodates up to $2 \mathrm{~mL}$ of sample. The method has been used in a SPINlab (5 T/0.8 K) for hyperpolarized water studies in a pig model [42], where dynamic angiography time series and perfusion maps were produced. The fluid path is already used in clinical studies [43] and it is reasonable to expect that the presented method can be translated into a sterile operation. The safety of the formulation needs to be established.

At maximum power, the DNP time constant was $86 \mathrm{~min}$, whereas the samples were polarized for $60 \mathrm{~min}$. Thus, the reported liquid state polarization could have been increased further by a factor two by longer polarization time. The polarization after $1 \mathrm{hr}$ showed a linear trend in the available power range (Figure 4), indicating that higher polarization after $1 \mathrm{hr}$ could be realized by increasing the microwave power above 55 $\mathrm{mW}$ through a shortening of the build-up time constant. The build-up time constant follows an inverse square-root of microwave power dependence, i.e. is inversely proportional to the microwave $B_{1}$ field. This is the expected dependence of the rate constant for both the solid-effect and thermal mixing [44]. The MW power at the entry to the cavity was measured to be approx. $5 \mathrm{~mW}$, but since we do not have details about the power distribution within the cavity, all data has been referenced to the source output power. 
[Type here]

The MW frequency modulation increased DNP efficiency for all tested samples. The $20 \mathrm{mM}$ water/glycerol $1: 1(\mathrm{w} / \mathrm{w})$ sample was tested for modulation amplitude of 25,50 , and $75 \mathrm{MHz}$, respectively, and $50 \mathrm{MHz}$ gave the largest DNP gain. $75 \mathrm{MHz}$ modulation amplitude was the optimal of the three for $30 \mathrm{mM}$ water/glycerol 1:1 (w/w) sample, whereas the $30 \mathrm{mM}$ water/DMSO- $\mathrm{d}_{6}$ 1:1 (w/w) sample polarized most efficiently at $125 \mathrm{MHz}$. Thus, both sample composition and radical concentration affect the frequency modulation performance. The modulation of the MW frequency is beneficial due to the inhomogeneous nature of the nitroxide EPR line at high magnetic field that restricts spectral diffusion. The inhomogeneity of the EPR line could probably have been overcome by increasing the nitroxide concentration further, but this may lead to shortening of $T_{1}$ in the liquid state and/or further loss in the dissolution.

Optimal MW frequencies were determined for several sample compositions at 6.7 T. Polarizers at other field strengths have other DNP frequency profiles, where optimal frequency modulation parameters are shifted. For polarizers without ${ }^{1} \mathrm{H}$ detection, the ${ }^{13} \mathrm{C}$ frequency sweeps can be used for localization of optimum EPR frequencies, and applied to protons due to the broad linewidth of the nitroxide radical. This is in principle how the SPINlab experiments were performed.

We have demonstrated that large volumes of hyperpolarized water can be produced with the fluid path technology. The $30 \mathrm{mM}$ water/glycerol 1:1 (w/w) sample polarized $30 \%$ better than the corresponding 20 $\mathrm{mM}$ sample, whereas the dissolved state polarization was $10 \%$ higher for the $20 \mathrm{mM}$ sample. The lower radical concentration in the dissolved state is the obvious reason. Degassing of the sample only increased the solid state polarization by $13 \%$, but increased liquid state polarization by $51 \%$. This is in good agreement with [24] where an approx. 50\% increase of the liquid state enhancement is observed after degassing.

Some liquid state polarization measurements showed large deviations. Only small variance in the solid state polarization was observed, which means that the dissolution process, extraction and sample transfer are the dominating sources of variability. The manual extraction process, where a funnel was shaken by hand, is inevitably a variable operation. The variation in the transfer time should be a minor contribution if the measured $T_{1}$ values are constant during the process. However, the relaxivity of the nitroxide is about the double in low field, and a shorter $T_{1}$ during the extraction and transfer should be expected. However, a more systematic variation in performance was also observed over time, possibly due to e.g. variation in microwave power delivery due to waveguide contamination.

Dissolving the frozen sample in heptane yielded low final polarizations. The low-density heptane places itself in the top in the dissolution medium syringe, and thus exits first. Thus, the sample was melted without being diluted until the aqueous phase appeared, causing a loss in polarization from relaxation by 
[Type here]

the high radical and proton concentration. Secondly, the fluid path plastic did not tolerate well the heated heptane and showed tendency of cracking. Instead, NFBME was determined to have TEMPO partition coefficient similar to that of heptane, and was tested with volume of $5,8,11 \mathrm{~mL}$, where the lower volume yielded the most controlled and efficient dissolution, and highest polarization in the liquid state.

NFBME volumes above $5 \mathrm{~mL}$ resulted in uncontrolled dissolution, where the exit tube was blown out of the receiver or the sample was spurted out of the funnel due to high pressure sample and gas from the exit tube. An extraction system with a fixed, stirred receiver or application of a filter at the exit tube may allow larger NFBME volume, resulting in faster, warmer dissolutions. In addition, this would allow larger sample sizes, so the full potential of the $2 \mathrm{~mL}$ vial could be utilized. The water/glassing agent ratio was not investigated. Smaller glassing agent volume may yield faster sample melting and lower radical concentration in the dissolved state, but the glassing ability of such sample should be verified first.

The radical removal may be optimized, e.g. by automatization of the extraction, which could also reduce the variance in the dissolved state polarization. Although radical quenching can be done efficiently, removal is preferred for in vivo studies and future clinical applications. Thus, filtering, reverse phase or ion exchange extraction of the radical may prove useful.

We did not observe relaxation times above $40 \mathrm{~s}$, as demonstrated in [24]. We believe that the decrease in temperature from dissolution to NMR acquisition is the main reason. In [24], the dissolved sample of $52^{\circ} \mathrm{C}$ was transferred in a preheated transfer line into a preheated NMR tube at $60^{\circ} \mathrm{C}$, whereas the $50{ }^{\circ} \mathrm{C}$ dissolved sample of the present study was cooled in the transferring syringe, injection line and NMR tube at room temperature. Since the aim of our study was to enable in vivo studies, the sample temperature should be conditioned to $30-35{ }^{\circ} \mathrm{C}$ at time of injection. The slightly longer $T_{1}$ at $3 \mathrm{~T}$ support this, as the larger sample volume used in these experiments will stay at the higher solvent exit temperature of the polarizer during measurement.

The liquid state NMR signal yielded robust polarization quantification, and the radiation damping time constant was demonstrated to give consistent measure of polarization.

The $T_{1}$ of the hyperpolarized samples in the liquid state are in the range 15-20 s, with uncertainty of the temperature and field dependence during transfer. With a dissolution, extraction and transfer time of approx. $25 \mathrm{~s}$, the relaxation loss amounts to approx. $70 \%\left(\mathrm{e}^{-25 \mathrm{~s} / 20 \mathrm{~s}}=0.3\right)$. This explains most of the polarization loss from the solid state $(P=70 \%)$ to the liquid state $(P=13 \%)$, but probably not entirely. Further optimization and automation of the radical removal and transfer may reduce this loss further, e.g. by 
[Type here]

sample injection systems. Similarly, the ${ }^{1} \mathrm{H}$ concentration may be further reduced to prolong $\mathrm{T}_{1}$ for the given transfer time. 
[Type here]

\section{Figure legends}

Figure 1: Build-up of the 1H NMR signal to thermal equilibrium for a $30 \mathrm{mM}$ TEMPO in water/glycerol 1:1 $(\mathrm{w} / \mathrm{w})$ sample at $6.7 \mathrm{~T}$ after subtraction of background signal. The $T_{1}$ was $583 \mathrm{~min}$.

Figure 2: Short MW frequency sweeps. a) $30 \mathrm{mM}$ TEMPO in water/glycerol 1:1 (w/w) with no frequency modulation (o) and modulation amplitude of $25 \mathrm{MHz}(+), 50 \mathrm{MHz}\left({ }^{*}\right)$ and $75 \mathrm{MHz}(\mathrm{x})$. b) $20 \mathrm{mM}$ TEMPO in water/glycerol 1:1 (w/w) with modulation amplitude of $25 \mathrm{MHz}(+), 50 \mathrm{MHz}\left({ }^{*}\right)$ and $75 \mathrm{MHz}$ (x). c) $30 \mathrm{mM}$ TEMPO in water/DMSO- $\mathrm{d}_{6} 1: 1(\mathrm{w} / \mathrm{w})$ with no frequency modulation (o) and modulation amplitude of 25 $\mathrm{MHz}(+), 50 \mathrm{MHz}(*), 75 \mathrm{MHz}(\mathrm{x}), 100 \mathrm{MHz}(\square), 125 \mathrm{MHz}(\diamond), 150 \mathrm{MHz}(\Delta)$ and $200 \mathrm{MHz}(\nabla)$. Modulation frequency $\mathrm{f}_{\bmod }=1 \mathrm{kHz}$.

Figure 3: Long MW frequency sweep without modulation. Final polarization (o) and DNP time constant ( $\square$ ) of build up for $30 \mathrm{mM}$ TEMPO in water/glycerol 1:1 (w/w) sample.

Figure 4: DNP power sweep of $20 \mathrm{mM}$ TEMPO in water/glycerol 1:1 (w/w) for microwave power 5 to 55 $\mathrm{mW}$ at $188.06 \mathrm{GHz}$ with modulation amplitude $50 \mathrm{MHz}$ and frequency $1 \mathrm{kHz}$. Polarization after $1 \mathrm{hr} \mathrm{DNP}(\Delta)$, asymptotical max polarization (0) with linear fit and DNP time constant estimated from a fit of $60 \mathrm{~min}$ polarization ( $\square$, build up at $5 \mathrm{~mW}$ was too slow to estimate time constant) with fit of inverse square root.

Figure 5: Short MW frequency sweep for $20 \mathrm{mM}$ TEMPO in water/glycerol 1:1 (w/w) at $60 \mathrm{~mW}(\square)$ and 30 $\mathrm{mW}$ MW power (o). $60 \mathrm{~s} \mathrm{MW}$ irradiation with frequency modulation of $\mathrm{a}_{\bmod }=50 \mathrm{MHz}$ and $\mathrm{f}_{\bmod }=1 \mathrm{kHz}$ was applied. The data confirms that build-up rate constant is independent of MW frequency.

Figure 6: a) Spectrum of hyperpolarized $30 \mathrm{mM}$ TEMPO in water/glycerol 1:1 (w/w) sample after dissolution. The small shoulder represents the glycerol protons. $50 \%$ linewidth of $1166 \mathrm{~Hz}$. b) Linear regression of linewidth of thermal spectra from samples of varying $\mathrm{H}_{2} \mathrm{O}: \mathrm{D}_{2} \mathrm{O}$ ratio.

Figure 7: Normalized NMR signal of hyperpolarized water sample (0) (30 mM TEMPO in water/glycerol 1:1 $(w / w))$ and build-up of relaxed sample at $9.4 T$ to validate $T_{1}(\square)$. The initial hyperpolarized signal is 3,761 times that at thermal equilibrium. The $T_{1}$ for the hyperpolarized sample was $17.0 \mathrm{~s}$, whereas the build-up to thermal equilibrium had a $T_{1}$ of $16.6 \mathrm{~s}$ (sample at room temperature). 
[Type here]

\section{Bibliography}

[1] J.H. Ardenkjaer-Larsen, B. Fridlund, A. Gram, G. Hansson, L. Hansson, M.H. Lerche, R. Servin, M. Thaning, K. Golman, Increase in signal-to-noise ratio of $>10,000$ times in liquid-state NMR., Proc. Natl. Acad. Sci. U. S. A. 100 (2003) 10158-10163. doi:10.1073/pnas.1733835100.

[2] S.E. Day, M.I. Kettunen, F.A. Gallagher, D.-E. Hu, M. Lerche, J. Wolber, K. Golman, J.H. ArdenkjaerLarsen, K.M. Brindle, Detecting tumor response to treatment using hyperpolarized $13 \mathrm{C}$ magnetic resonance imaging and spectroscopy., Nat. Med. 13 (2007) 1382-1387. doi:10.1038/nm1650.

[3] M.J. Albers, R. Bok, A.P. Chen, C.H. Cunningham, M.L. Zierhut, V.Y. Zhang, S.J. Kohler, J. Tropp, R.E. Hurd, Y.F. Yen, S.J. Nelson, D.B. Vigneron, J. Kurhanewicz, Hyperpolarized 13C lactate, pyruvate, and alanine: Noninvasive biomarkers for prostate cancer detection and grading, Cancer Res. 68 (2008) 8607-8615. doi:10.1158/0008-5472.CAN-08-0749.

[4] H. Gutte, A.E. Hansen, H.H. Johannesen, A.E. Clemmensen, J.H. Ardenkjær-Larsen, C. Haagen, A. Kjær, The use of dynamic nuclear polarization 13 C-pyruvate MRS in cancer, Am. J. Nucl. Med. Mol. Imaging. 5 (2015) 548-560.

[5] R.E. Hurd, Y.-F. Yen, J. Tropp, A. Pfefferbaum, D.M. Spielman, D. Mayer, Cerebral dynamics and metabolism of hyperpolarized [1-(13)C]pyruvate using time-resolved MR spectroscopic imaging., J. Cereb. Blood Flow Metab. 30 (2010) 1734-1741. doi:10.1038/jcbfm.2010.93.

[6] C. Laustsen, K. Lipsø, J.A. Ostergaard, R. Nørregaard, A. Flyvbjerg, M. Pedersen, F. Palm, J.H. Ardenkjær-Larsen, Insufficient insulin administration to diabetic rats increases substrate utilization and maintains lactate production in the kidney, Physiol. Rep. 2 (2014) e12233-e12233. doi:10.14814/phy2.12233.

[7] L.E. Olsson, C.M. Chai, O. Axelsson, M. Karlsson, K. Golman, J.S. Petersson, MR coronary angiography in pigs with intraarterial injections of a hyperpolarized 13C substance, Magn. Reson. Med. 55 (2006) 731-737. doi:10.1002/mrm.20847.

[8] K. Golman, J.H. Ardenkjær-Larsen, J. Svensson, O. Axelsson, G. Hansson, L. Hansson, H. Jóhannesson, I. Leunbach, S. Månsson, J.S. Petersson, G. Pettersson, R. Servin, L.-G. Wistrand, 13C-Angiography, Acad. Radiol. 9 (2002) 507-510. doi:10.1016/S1076-6332(03)80278-7.

[9] K.W. Lipsø, P. Magnusson, J.H. Ardenkjær-Larsen, Hyperpolarized 13 C MR Angiography, Curr. Pharm. Des. 22 (2016) 90-95. doi:10.2174/1381612822666151109112415.

[10] S. Bowen, C. Hilty, Time-resolved dynamic nuclear polarization enhanced NMR spectroscopy, Angew. Chemie - Int. Ed. 47 (2008) 5235-5237. doi:10.1002/anie.200801492. 
[11] C. Harrison, C. Yang, A. Jindal, R.J. Deberardinis, M.A. Hooshyar, M. Merritt, A. Dean Sherry, C.R. Malloy, Comparison of kinetic models for analysis of pyruvate-to-lactate exchange by hyperpolarized 13C NMR, NMR Biomed. 25 (2012) 1286-1294. doi:10.1002/nbm.2801.

[12] H. Allouche-Arnon, A. Gamliel, C.M. Barzilay, R. Nalbandian, J.M. Gomori, M. Karlsson, M.H. Lerche, R. Katz-Brull, A hyperpolarized choline molecular probe for monitoring acetylcholine synthesis, Contrast Media Mol. Imaging. 6 (2011) 139-147. doi:10.1002/cmmi.418.

[13] M.J. Bayro, G.T. Debelouchina, M.T. Eddy, N.R. Birkett, C.E. Macphee, M. Rosay, W.E. Maas, C.M. Dobson, R.G. Griffin, Intermolecular Structure Determination of Amyloid Fibrils with Magic-Angle Spinning and Dynamic Nuclear Polarization NMR, J. Am. Chem. Soc. 133 (2011) 13967-13974.

[14] A. Potapov, W.M. Yau, R. Tycko, Dynamic nuclear polarization-enhanced 13C NMR spectroscopy of static biological solids, J. Magn. Reson. 231 (2013) 5-14. doi:10.1016/j.jmr.2013.02.011.

[15] P. Mark D. Lingwood, B. Ting Ann Siaw, P. Napapon Sailasuta, M. Osama A. Abulseoud, M. Henry R. Chan, M. Brian D. Ross, P. Pratip Bhattacharya, P. Songi Han, Hyperpolarized Water as an MR Imaging Contrast Agent: Feasibility of in Vivo Imaging in a Rat Model, Radiology. 265 (2012) 418425. doi:10.1148/radiol.12111804.

[16] R.E. Lenkinski, Science to Practice: Can Hyperpolarized Water Be Used to Enhance MR Angiography and Flow Measurement?, Radiology. 265 (2012) 325-326. doi:10.1148/radiol.12121642.

[17] E.R. McCarney, B.D. Armstrong, M.D. Lingwood, S. Han, Hyperpolarized water as an authentic magnetic resonance imaging contrast agent., Proc. Natl. Acad. Sci. U. S. A. 104 (2007) 1754-1759. doi:10.1073/pnas.0610540104.

[18] M.D. Lingwood, T.A. Siaw, N. Sailasuta, B.D. Ross, P. Bhattacharya, S. Han, Continuous flow Overhauser dynamic nuclear polarization of water in the fringe field of a clinical magnetic resonance imaging system for authentic image contrast, J. Magn. Reson. 205 (2010) 247-254. doi:10.1016/j.jmr.2010.05.008.

[19] J.H. Ardenkjaer-Larsen, C. Laustsen, S. Bowen, R. Rizi, Hyperpolarized H2O MR angiography, Magn. Reson. Med. 71 (2014) 50-56. doi:10.1002/mrm.25033.

[20] B.D. Armstrong, S. Han, Overhauser dynamic nuclear polarization to study local water dynamics., J. Am. Chem. Soc. 131 (2009) 4641-4647. doi:10.1021/ja809259q.

[21] J.-H. Ardenkjaer-Larsen, G.S. Boebinger, A. Comment, S. Duckett, A.S. Edison, F. Engelke, C. Griesinger, R.G. Griffin, C. Hilty, H. Maeda, G. Parigi, T. Prisner, E. Ravera, J. van Bentum, S. Vega, A. Webb, C. Luchinat, H. Schwalbe, L. Frydman, Facing and Overcoming Sensitivity Challenges in 
Biomolecular NMR Spectroscopy, Angew. Chemie Int. Ed. 54 (2015) 9162-9185. doi:10.1002/anie.201410653.

[22] T. Harris, O. Szekely, L. Frydman, On the potential of Hyperpolarized Water in Biomolecular NMR Studies, J. Phys. Chem. B. 118 (2014) 3281-3290. doi:10.1021/jp4102916.

[23] Q. Chappuis, J. Milani, B. Vuichoud, A. Bornet, A.D. Gossert, G. Bodenhausen, S. Jannin, Hyperpolarized Water to Study Protein-Ligand Interactions, J. Phys. Chem. Lett. 6 (2015) 16741678. doi:10.1021/acs.jpclett.5b00403.

[24] G. Olsen, E. Markhasin, O. Szekely, C. Bretschneider, L. Frydman, Optimizing water hyperpolarization and dissolution for sensitivity-enhanced 2D biomolecular NMR, J. Magn. Reson. 264 (2016) 49-58. doi:10.1016/j.jmr.2016.01.005.

[25] B. Vuichoud, A. Bornet, F. de Nanteuil, J. Milani, E. Canet, X. Ji, P. Miéville, E. Weber, D. Kurzbach, A. Flamm, R. Konrat, A.D. Gossert, S. Jannin, G. Bodenhausen, Filterable Agents for Hyperpolarization of Water, Metabolites, and Proteins, Chem. Eur. J. 22 (2016) 1521-3765. doi:10.1002/chem.201602506.

[26] J.H. Ardenkjaer-Larsen, A.M. Leach, N. Clarke, J. Urbahn, D. Anderson, T.W. Skloss, Dynamic nuclear polarization polarizer for sterile use intent, NMR Biomed. 24 (2011) 927-932. doi:10.1002/nbm.1682.

[27] A. Bornet, J. Milani, B. Vuichoud, A.J. Perez Linde, G. Bodenhausen, S. Jannin, Microwave Frequency Modulation to Enhance Dissolution Dynamic Nuclear Polarization, Chem. Phys. Lett. 602 (2014) 6367. doi:10.1016/j.cplett.2014.04.013.

[28] Y. Hovav, A. Feintuch, S. Vega, D. Goldfarb, Dynamic nuclear polarization using frequency modulation at 3.34 T, J. Magn. Reson. 238 (2014) 94-105. doi:10.1016/j.jmr.2013.10.025.

[29] V. Weis, M. Bennati, M. Rosay, J.A. Bryant, R.G. Griffin, High-field DNP and ENDOR with a novel multiple-frequency resonance structure, J. Magn. Reson. 140 (1999) 293-299. doi:10.1006/jmre.1999.1841.

[30] P. Höfer, G. Parigi, C. Luchinat, P. Carl, G. Guthausen, M. Reese, T. Carlomagno, C. Griesinger, M. Bennati, Field dependent dynamic nuclear polarization with radicals in aqueous solution, J. Am. Chem. Soc. 130 (2008) 3254-3255. doi:10.1021/ja0783207.

[31] P. Miéville, P. Ahuja, R. Sarkar, S. Jannin, P.R. Vasos, S. Gerber-Lemaire, M. Mishkovsky, A. Comment, R. Gruetter, O. Ouari, P. Tordo, G. Bodenhausen, Scavenging free radicals to preserve enhancement and extend relaxation times in NMR using dynamic nuclear polarization, Angew. 
Chemie - Int. Ed. 49 (2010) 6182-6185. doi:10.1002/anie.201000934.

[32] T. Harris, C. Bretschneider, L. Frydman, Dissolution DNP NMR with solvent mixtures: Substrate concentration and radical extraction, J. Magn. Reson. 211 (2011) 96-100. doi:10.1016/j.jmr.2011.04.001.

[33] J.T. Anderson, W. A.; Arnold, Proton Relaxation Times in H2O-D2O Mixtures, Phys. Rev. 101 (1956) 511-512. doi:10.1103/PhysRev.101.511.

[34] H.Y. Simpson, J. H.; Carr, Diffusion and Nuclear Spin Relaxation in Water, Phys. Rev. 111 (1958) 1201-1202. doi:10.1103/PhysRev.111.1201.

[35] N. Nestle, T. Baumann, R. Niessner, Oxygen determination in oxygen-supersaturated drinking waters by NMR relaxometry, Water Res. 37 (2003) 3361-3366. doi:10.1016/S0043-1354(03)00211-2.

[36] R. Battino, T.R. Rettich, T. Tominaga, The Solubility of Oxygen and Ozone in Liquids, J. Phys. Chem. Ref. Data. 12 (1983) 163-178. doi:10.1063/1.555680.

[37] Michael G. Costello, R.M. Flynn, J.G. Owens, 3M Company, Fluoroethers and Fluoroaminies, KirkOthmer Encyclopedia of Chemical Technology, 5th ed., John Wiley \& Sons, 2005. doi:10.1002/0471238961.

[38] V. V. Krishnan, N. Murali, Radiation damping in modern NMR experiments: Progress and challenges, Prog. Nucl. Magn. Reson. Spectrosc. 68 (2013) 41-57. doi:10.1016/j.pnmrs.2012.06.001.

[39] J. Tropp, M. Van Criekinge, Radiation damping and reciprocity in nuclear magnetic resonance: The replacement of the filling factor, J. Magn. Reson. 206 (2010) 161-167. doi:10.1016/j.jmr.2010.06.001.

[40] O. Rybalko, S. Bowen, V. Zhurbenko, J.H. Ardenkjær-Larsen, Waveguide transition with vacuum window for multiband dynamic nuclear polarization systems, Rev. Sci. Instrum. 87 (2016) 54705. doi:10.1063/1.4948294.

[41] R.M. Malinowski, K.W. Lips $\varnothing$, M.H. Lerche, J.H. Ardenkjær-larsen, Dissolution Dynamic Nuclear Polarization capability study with fluid path, J. Magn. Reson. 272 (2016) 141-146. doi:10.1016/j.jmr.2016.09.015.

[42] K.W. Lipsø, E.S. Szocska Hansen, R. Tougaard, C. Laustsen, J.H. Ardenkjær-Larsen, Renal MR Angiography and Perfusion in the Pig using Hyperpolarized Water, Magn. Reson. Med. In press (2016). doi:10.1002/mrm.26478.

[43] C.H. Cunningham, J.Y. Lau, A.P. Chen, B.J. Geraghty, W.J. Perks, I. Roifman, G.A. Wright, K.A. 
[Type here]

Connelly, Hyperpolarized 13 C Metabolic MRI of the Human Heart: Initial Experience, Circ. Res. 119 (2016) 1177-1182. doi:10.1161/CIRCRESAHA.116.309769.

[44] T. Wenckebach, Essentials of Dynamic Nuclear Polarization, Spindrift Publications, The Netherlands, 9 May 2016. ISBN 9789075541182. 